Ann. Biol. anim. Bioch. Biophys., 1979, 19 (1 A), 79-90.

\title{
Excrétion biliaire chez le porc : niveau et réponse au repas
}

\author{
par Catherine JUSTE, T. CORRING, Ph. BREANT \\ avec la collaboration technique de Georgette FREDOU, A. ROGER, A. Van HECKE \\ Laboratoire de Physiologie de la Nutrition \\ I.N.R.A., 78350 jouy-en-josas.
}

Summary. Bile excretion in pig. Magnitude and response to meal intake.

A technique for permanent fistulation of the bile duct, associated with a permanent fistulation of the duodenum allowing continuous reintroduction of the collected bile, was used to investigate the level and daily kinetics of biliary excretion in the conscious pig. Seven Large-White pigs of $45 \mathrm{~kg}$ live weight were fistulated. Volumes and total excreted bile salts were measured in hourly samples. Results show that within 24 hrs. the volume of the collected bile was about $2100 \mathrm{ml}$ and the total amount of bile salts about $37 \mathrm{~g}$. The bile excretion (volume and bile salts) was minimum before the morning meal (9:00 a. m.) and the total amount of the excreted bile salts immediately increased after diet intake. Within 2 hrs. after the morning and afternoon meals, values were about 300 p. 100 higher than those determined at 9:00 a. m. This increase was essentially the result of the excretion of a highly concentrated bile (200 to 300 p. 100 more than the values at 9:00 a. m.). On the other hand, the bile volumes were only affected 2 to $3 \mathrm{hrs}$. after diet intake.

\section{Introduction.}

L'implication de la sécrétion biliaire dans la digestion intraluminale est connue de longue date (Bergstrom et Borgstrom, 1955 ; Hofmann, 1964) et concerne essentiellement la dégradation des graisses alimentaires ef l'absorption des acides gras libérés. Chez le Porc, la digestibilité apparente des lipides totaux diminue de 52 p. 100 environ, en l'absence de bile dans la lumière intestinale (Corring et al., données non publiées).

Les premières observations visant à décrire la rythmicité de l'activité hépatique chez le Lapin (Forsgren, 1928) chez la Souris (Holmgren, 1931), chez le Rat (Holmquist, 1931) et chez l'Homme (Martin et Sciclounoff, 1934) n'onł pu mettre en évidence un rapport direct entre la sécrétion biliaire et la prise de nourrifure. D'après ces études, la sécrétion de la bile et son excrétion obéissent à un rythme circadien, mais les fluctuations journalières observées dépendent moins de l'heure des repas que de l'alternance du jour et de la nuit. Par la suite, des méthodes d'investigation plus élaborées, telles que la fistulation permanente et totale du canal cholédoque chez le Chien (Jones et Grossman, 1969), le dosage d'acides biliaires dans le tissu hépatique 
du Rat (Bortz et Steele, 1973), ou encore le dosage des acides biliaires sanguins chez l'Homme (La Russo et al., 1974) ont permis de montrer que le niveau de la sécrétion biliaire variait en fonction des heures de repas. Enfin, très récemment, la même relation bile-alimentation a été envisagée chez le Porc, dans deux travaux différents (Laplace ef Ouaissi, 1977 ; Sambrook, 1978). Selon Laplace ef Ouaissi (1977), l'ingestion de nourriture ne semble pas avoir un effet direct sur le débit biliaire. Sambrook (1978), en revanche, démontre l'existence d'un tel effeł et souligne qu'il serait lié à la nature du régime.

Dans ces deux dernières études, seul le débit de la sécrétion biliaire a été considéré. Etant donné l'importance des sels biliaires dans la digestion intraluminale, il nous est apparu essentiel de décrire chez le Porc, la cinétique des concentrations ef des quantités de sels biliaires totaux excrétés sur plusieurs nycthémères.

\section{Matériel et méthodes.}

\section{Animaux.}

Sept porcs mâles castrés de race Large White et de poids vif compris entre 40 et $50 \mathrm{~kg}$ ont éfé utilisés. Huit jours avant l'opération, ils commencent à recevoir le régime expérimenral dont la composition est donnée dans le tableau 1. Les animaux reçoivent deux repas de $800 \mathrm{~g}$ chacun par $24 \mathrm{~h}$, à $9 \mathrm{~h}$ ef $16 \mathrm{~h}$.

\section{TABLEAU 1}

Composition de l'aliment expérimental (en p. 100 de la matière fraîche)

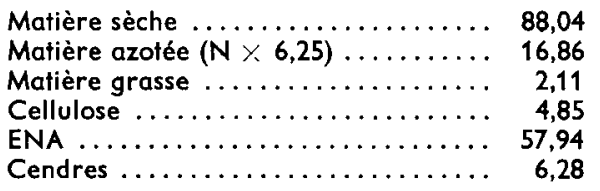

Technique opératoire.

Le principe consiste à dévier la totalité de la sécrétion biliaire à l'extérieur de l'animal, afin de mesurer les volumes excrétés en fonction du temps et de doser les quantités de sels biliaires présents à chaque instanr. Après échantillonnage, la bile recueillie est réintroduite au niveau du duodénum.

Préparation des cathéters. - On utilise des cathéters en silastic $\left(^{1}\right)$ pour les fistules cholédocienne et duodénale. Pour le cholédoque, les diamètres intérieurs ef extérieurs des canules varient suivant sa taille $(\varnothing$ intérieur : $2,64 \mathrm{~mm} ; 3,18 \mathrm{~mm} ; 4,76 \mathrm{~mm}$ $\varnothing$ extérieur : 4,88 $\mathrm{mm} ; 6,35 \mathrm{~mm} ; 7,94 \mathrm{~mm}$ ). Pour le duodénum, on utilise un cathéter de $\varnothing$ intérieur : $2,64 \mathrm{~mm}$ et de $\varnothing$ extérieur : $4,88 \mathrm{~mm}$. Ces cathéłers ont une longueur

(1) Dow Corning Corporation medical Products, Midland, Michigan, 48640 USA. 
de $50 \mathrm{~cm}$ et sont munis à une extrémité de deux bagues en silicone, espacées de $5 \mathrm{~cm}$ environ. Avant utilisation, les cathéters sont stérilisés dans un aułoclave à $160^{\circ} \mathrm{C}$ pendant une heure.

Mise en place des cathéters.

- 1er temps opératoire : La peau et les tissus sous-cutanés sont incisés sur une longueur de $20 \mathrm{~cm}$ environ, parallèlement et légèrement en arrière de la dernière cote flottante. Les différentes couches musculaires sont écartées dans le sens des fibres et le péritoine est ouvert selon l'axe d'incision de la peau.

- $2^{\text {e }}$ temps opératoire : Les lèvres de l'incision sont écartées à l'aide d'un écarteur de Gosset. L'estomac est saisi, ramené sans traction vers l'ouverture et maintenu à l'extérieur de la cavité abdominale par une pince à enclenchement. Le canal cholédoque, débouchant en partie haute du duodénum, à proximité du pylore, est localisé, dégagé du mésentère sur $1 \mathrm{~cm}$ et environ à $0,5 \mathrm{~cm}$ en amont du sphincter d'Oddi.

- 3e temps opératoire : La partie dénudée du canal est incisée transversalement, sur une demi-circonférence, à l'aide de petits ciseaux aigus. La bile apparaît spontanément au niveau de l'incision. Le cathéter est introduit avec précaution en direction du foie et sur une longueur de 2 à $3 \mathrm{~cm}$. La première bague est engagée dans le canal. Une ligature en aval de cette bague permet de refermer le canal autour du cathéter. La canule est fixée sur la paroi duodénale, au niveau de la deuxième bague, par une boucle auto-serreuse.

Le cathéter intestinal est ensuite introduit dans le duodénum, à proximité de l'abouchement naturel du cholédoque. La première bague est engagée dans la lumière intestinale et la paroi est refermée immédiatement en aval de la bague par un point en bourse auto-serreur. La canule est fixée à la surface du duodénum par une boucle auto-serreuse au niveau de la deuxième bague.

- 4e temps opératoire : Deux orifices pratiqués dans le flanc droit par ponction au bistouri, permettent la sortie des deux cathéters à l'extérieur de la cavité abdominale. La partie du duodénum où sont fixées les deux canules est ensuite ramenée contre le péritoine, afin d'éviter qu'une anse intestinale ne vienne ultérieurement s'étrangler autour des tubes en silastic (volvulus intestinal).

La plaie abdominale est refermée par suture des différents plans qui constituent la paroi. Les cathéters sont fixés à la peau à l'aide de nœuds auto-serreurs.

Soins post-opératoires. - Les infections sont prévenues à l'aide de trois injections intramusculaires de pénicilline-retard (1 million UI) ef de streptomycine $(1 \mathrm{~g})$ à $24 \mathrm{~h}$ d'intervalle après l'opération.

\section{Schéma expérimental.}

Utilisation des animaux. - Après opération, les animaux sonł placés en cage individuelle, type cage à métabolisme. Ils sont nourris 48 h après l'intervention chirurgicale. La ration journalière est progressivement augmentée pour atteindre $800 \mathrm{~g}$ en matière fraîche par repas, au bout d'une semaine.

Les collectes ef réintroductions de bile commencent dès le premier jour, mais 
les enregistrements volumétriques ef les dosages de sels biliaires ne débutent pas avant le rétablissement complet des animaux, soit 10 à 15 jours après l'opération.

Utilisation des fistules. - La bile est recueillie 24 h sur 24 sur un collecteur de fractions dont la rotation intervient toutes les $10 \mathrm{mn}$. Les relevés volumétriques sont réalisés à $9 \mathrm{~h}, 12 \mathrm{~h}$ et $17 \mathrm{~h}$ ef les quantités de bile excrétée sont exprimées en $\mathrm{ml} / \mathrm{h}$. Il a été prélevé environ $5 \mathrm{ml}$ de bile par échantillon horaire pour la détermination des concentrations en sels biliaires totaux. L'ensemble des prélèvements représente en moyenne 5 p. 100 du volume de bile excrétée en $24 \mathrm{~h}$. Après chaque relevé (à $9 \mathrm{~h}$, $12 \mathrm{~h}$ et $17 \mathrm{~h}$ ), la bile est destinée à réintroduction. Celle-ci est assurée par une pompe à régulation électronique dont le débit est réglé manuellement en fonction des heures de la journée. Des observations préliminaires ont montré que l'excrétion des sels biliaires était maximale pendant les périodes postprandiales, ce qui nous a conduit à augmenter durant ces périodes le débit de la pompe de réintroduction.

Détermination enzymatique des sels biliaires totaux. - La technique de détermination des sels biliaires totaux est inspirée de la méthode décrite par Talalay et Dobson (1953) et Hurlock ef Talalay $(1956,1957)$ et repose sur un dosage enzymatique ( $3 \alpha$-hydroxystéroïde-déhydrogénase) avec lectures spectrophorométriques à $340 \mathrm{~nm}$.

\section{Résultats.}

Tous les animaux opérés ont été observés pendant une durée de $15 \pm 3$ jours. Dans tous les cas, le rejet du cathéter de collecte a entraîné l'arrêt de l'expérimentation 25 à 30 jours après l'opération. En outre, de nombreuses difficultés techniques liées à l'utilisation du collecteur de fractions (arrêts, dérèglement de la rotation, etc...) ont provoqué d'importantes pertes d'échantillons (principalement au cours de la nuit) et de fait ont conduit à l'élimination d'un grand nombre de journées expé-

TABLEAU 2

Niveau de l'excrétion biliaire journalière chez le porc

\begin{tabular}{|c|c|c|c|c|c|c|c|c|c|c|}
\hline \multirow[t]{2}{*}{ Porc } & \multirow[t]{2}{*}{$\begin{array}{l}\text { Nombre de } \\
\text { journées } \\
\text { d'obser- } \\
\text { vation }\end{array}$} & \multicolumn{3}{|c|}{$\begin{array}{l}\text { Volume total } \\
\text { moyen de bile/ } \\
24 \mathrm{~h}(\mathrm{ml} / 24 \mathrm{~h})\end{array}$} & \multicolumn{3}{|c|}{$\begin{array}{c}\text { Concentration } \\
\text { horaire } \\
\text { moyenne } \\
\text { calculée s } / 24 \mathrm{~h} \\
(\mathrm{mg} / \mathrm{ml})\end{array}$} & \multicolumn{3}{|c|}{$\begin{array}{c}\text { Quantité } \\
\text { moyenne } \\
\text { de sels biliaires } \\
\text { totaux } / 24 \mathrm{~h} \\
(\mathrm{~g} / 24 \mathrm{~h})\end{array}$} \\
\hline & & $\bar{X}\left({ }^{1}\right)$ & $s \bar{X}\left({ }^{2}\right)$ & $C V\left({ }^{3}\right)$ & $\bar{x}$ & $s \bar{X}$ & $\mathrm{CV}$ & $\bar{x}$ & $\overline{S X}$ & CV \\
\hline $\begin{array}{l}1 \\
2 \\
3 \\
4 \\
5 \\
6 \\
7\end{array}$ & $\begin{array}{l}7 \\
5 \\
4 \\
3 \\
5 \\
6 \\
3\end{array}$ & $\begin{array}{ll}2 & 020 \\
2 & 305 \\
2 & 345 \\
2 & 210 \\
2 & 260 \\
1 & 235 \\
1 & 890\end{array}$ & $\begin{array}{r}100 \\
180 \\
110 \\
355 \\
100 \\
65 \\
175\end{array}$ & $\begin{array}{l}10 \\
15 \\
11 \\
23 \\
12 \\
10 \\
18\end{array}$ & $\begin{array}{r}9,5 \\
19,5 \\
17,7 \\
21,2 \\
27,6 \\
9,1 \\
8,5\end{array}$ & $\begin{array}{l}1,8 \\
2,1 \\
0,6 \\
0,9 \\
1,1 \\
0,8 \\
1,3\end{array}$ & $\begin{array}{r}38 \\
21 \\
7 \\
6 \\
11 \\
17 \\
30\end{array}$ & $\begin{array}{l}18,6 \\
44,2 \\
41,3 \\
47,3 \\
62,4 \\
11,1 \\
15,9\end{array}$ & $\begin{array}{l}3,3 \\
2,5 \\
3,2 \\
4,8 \\
2,6 \\
1,3 \\
3,5\end{array}$ & $\begin{array}{l}35 \\
11 \\
17 \\
14 \\
12 \\
23 \\
44\end{array}$ \\
\hline Total & 33 & 2065 & 79 & 21 & 17,4 & 1,4 & 46 & 37,4 & 3,6 & 55 \\
\hline
\end{tabular}

(1) Moyenne ; $\left({ }^{2}\right)$ Ecart-type de la moyenne ; $\left.{ }^{3}\right)$ Coefficient de variation en p. 100. 
rimentales complètes. Sur l'ensemble des journées d'observation, seules 33 d'entre elles ont été retenues. Par contre, les données horaires concernant les réponses aux repas sont plus nombreuses (cf. tabl. $3,4,5$ ).

Niveau journalier de l'excrétion biliaire. - Les résultats (tabl. 2) montrent que, pour l'ensemble des animaux, le volume total moyen de bile émise par $24 \mathrm{~h}$ est de $2065 \mathrm{ml}$, la concentration horaire moyenne de $17,4 \mathrm{mg} / \mathrm{ml}$ ef la quantité de sels biliaires totaux excrétés par $24 \mathrm{~h}$ de $37,4 \mathrm{~g}$. Les coefficients de variation de ces résultats traduisent des différences individuelles atteignant environ 50 p. 100 pour les concentrations ef quantités de sels biliaires. Comparativement, la variabilité des quantités journalières de bile émise par l'ensemble des animaux est beaucoup plus faible (21 p. 100).

Les coefficients de variation des moyennes individuelles se rapportant aux trois critères précédents (volume, concentration et quantité de sels biliaires) traduisent des différences d'un nycthémère à l'autre moins importantes que celles observées entre animaux sur l'ensemble des journées d'expérimentation. Ceci souligne la qualité des prélèvements, et montre que l'écoulement gravitaire de la bile, lié à la technique de fistulation utilisée, n'a eu aucune incidence dépressive ou stimulante sur les phénomènes secrétoires.

Réponse au repas. - Les fortes variations individuelles portant sur les moyennes quotidiennes se sont inévitablement retrouvées tout au long des heures de la journée.

\section{TABLEAU 3}

Éfude de l'excrétion biliaire chez le porc

Variations des quantités de bile émise (en pourcentage des valeurs obtenues à $9 \mathrm{~h}$ ).

Amplitude des maxima et du minimum, en fonction des heures de distribution des repas ( $9 \mathrm{~h}$ ef $16 \mathrm{~h}$ )

\begin{tabular}{|c|c|c|c|c|c|c|c|c|c|c|c|c|c|}
\hline \multirow{3}{*}{ Porc } & \multicolumn{4}{|c|}{ 1er maximum } & \multicolumn{4}{|c|}{ Minimum } & \multicolumn{4}{|c|}{$2^{\mathrm{e}}$ maximum } & \multirow{3}{*}{$\begin{array}{c}\text { Moyennes } \\
\text { observées } \\
\text { à } 9 \mathrm{~h} \\
\text { (base 100) } \\
\mathrm{ml} / \mathrm{h}\end{array}$} \\
\hline & \multirow{2}{*}{$\begin{array}{c}\text { Heure } \\
\text { do } \\
\text { jour }\end{array}$} & \multicolumn{3}{|c|}{ Volumes de bile } & \multirow{2}{*}{$\begin{array}{c}\text { Heure } \\
\text { du } \\
\text { jour }\end{array}$} & \multicolumn{3}{|c|}{ Volumes de bile } & \multirow{2}{*}{$\begin{array}{c}\text { Heure } \\
\text { du } \\
\text { jour }\end{array}$} & \multicolumn{3}{|c|}{ Volumes de bile } & \\
\hline & & $\bar{x}\left({ }^{1}\right)$ & $s \bar{X}\left({ }^{2}\right)$ & $C V\left({ }^{3}\right)$ & & $\bar{x}$ & $s \bar{x}$ & $\mathrm{CV}$ & & $\vec{x}$ & $\overline{s X}$ & $\mathrm{CV}$ & \\
\hline 1 & $\begin{array}{l}15 \mathrm{~h} \\
(10)\end{array}$ & 209 & 33 & 54 & $\begin{array}{l}19 \mathrm{~h} \\
(10)\end{array}$ & 141 & 26 & 62 & $\begin{array}{c}23 \mathrm{~h} \\
(7)\end{array}$ & 208 & 63 & 79 & 58 \\
\hline 2 & $15 \mathrm{~h}$ & 199 & 18 & 30 & $22 \mathrm{~h}$ & 128 & 20 & 46 & $\begin{array}{c}23 \mathrm{~h} \\
\text { (8) }\end{array}$ & 173 & 18 & 30 & 39 \\
\hline 3 & $\begin{array}{l}14 \mathrm{~h} \\
\text { (11) }\end{array}$ & 260 & 24 & 31 & $\begin{array}{l}18 \mathrm{~h} \\
(10)\end{array}$ & 158 & 15 & 30 & $\begin{array}{c}24 \mathrm{~h} \\
\text { (5) }\end{array}$ & 239 & 26 & 25 & 55 \\
\hline 4 & $\begin{array}{l}14 \mathrm{~h} \\
\text { (4) }\end{array}$ & 201 & 28 & 28 & $\begin{array}{l}17 \mathrm{~h} \\
\text { (3) }\end{array}$ & 116 & 3 & 4 & $\begin{array}{c}19 \mathrm{~h} \\
\text { (3) }\end{array}$ & 184 & 23 & 22 & 57 \\
\hline 5 & $\begin{array}{c}14 \mathrm{~h} \\
\text { (9) }\end{array}$ & 197 & 37 & 56 & $\begin{array}{c}20 \mathrm{~h} \\
(8)\end{array}$ & 145 & 22 & 43 & $\begin{array}{c}23 \mathrm{~h} \\
(8)\end{array}$ & 182 & 34 & 52 & 69 \\
\hline 6 & $\begin{array}{c}16 \mathrm{~h} \\
(6)\end{array}$ & 204 & 27 & 33 & $\begin{array}{c}18 \mathrm{~h} \\
\text { (6) }\end{array}$ & 122 & 9 & 18 & $\begin{array}{c}22 \mathrm{~h} \\
(6)\end{array}$ & 198 & 14 & 18 & 32 \\
\hline 7 & $\begin{array}{c}16 \mathrm{~h} \\
(6)\end{array}$ & 182 & 15 & 20 & $\begin{array}{c}18 \mathrm{~h} \\
(6)\end{array}$ & 83 & 8 & 23 & $\begin{array}{c}24 \mathrm{~h} \\
(6)\end{array}$ & 168 & 15 & 22 & 61 \\
\hline
\end{tabular}

(1) Moyenne ; ( ${ }^{2}$ ) Ecart-type de la moyenne ; $\left(^{3}\right)$ Coefficient de variation.

( ) Nombre d'observations effectuées pour un même animal à l'heure indiquée. 
De ce fait, l'établissement de moyennes horaires pour l'ensemble des porcs et nycthémères confondus aurait abouti au nivellement de l'évolution journalière de la sécrétion biliaire. Il est donc apparu nécessaire de considérer les animaux individuellement et de rapporter les enregistrements horaires en p. 100 des valeurs observées à $9 \mathrm{~h}$, avant la distribution du premier repas.

Cette expression, illustrée par la figure 1, a facilité la comparaison entre animaux et a permis de décrire l'évolution de la sécrétion de la bile ef celle de l'excrétion des sels biliaires.

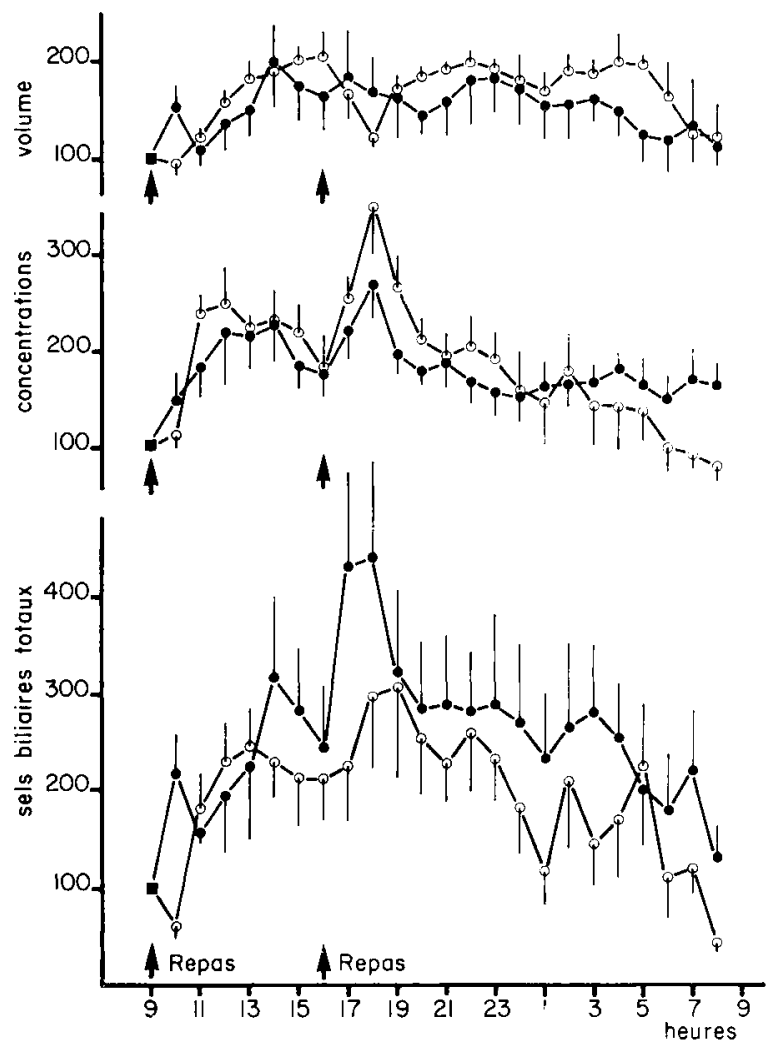

FIG. 1. - Evolution ou cours du nycthémère des volumes de bile émise, des concentrations en sels biliaires ef des quantités de sels biliaires totaux excrétés, exprimés en pourcentages des valeurs correspondantes mesurées à $9 \mathrm{~h}$.

Courbes moyennes, avec écarts-types de la valeur horaire moyenne.

$\bullet$ Pore 5

$\circ$ - Porc 6

1) Sécrétion de la bile. - Bien que les débits biliaires mesurés à une heure donnée diffèrent d'un jour sur l'autre chez le même animal (tabl.3), le même profil général s'observe pour les 7 porcs expérimentés ef se caractérise par deux pics secrétoires séparés par une période dépressive. 
Pendant les $2 \mathrm{~h}$ consécutives au premier repas, le volume biliaire oscille autour de la valeur 100, prise à $9 \mathrm{~h}$. La quantité de bile excrétée durant cette période est, selon les animaux, inférieure (cas des porcs 1 et 7), sensiblement égale (cas des porcs 2 et-6) ou supérieure (cas des porcs 3,4 et 5) à celle recueillie dans le même temps, avant le repas. A partir de la $3^{2}$ heure postprandiale, le débit biliaire augmente de façon importante pour tous les animaux, et atteint un maximum entre $14 \mathrm{~h}$ et $16 \mathrm{~h}$ selon les cas (200 à 250 p. 100 environ des valeurs observées à 9 h).

L'excrétion de bile diminue ensuite chez tous les animaux ef l'ingestion du deuxième repas n'affecte pas immédiatement cette diminution. On observe une nouvelle augmentation des volumes de bile émise seulement 2 à 6 h après cette dernière prise de nourriture. Les maxima alors observés atteignent en moyenne 200 p. 100 des valeurs prises à $9 \mathrm{~h}$.

La fin du nycthémère est marquée par un retour progressif des débits biliaires à des valeurs minimales entre $6 \mathrm{~h}$ et $9 \mathrm{~h}$ du matin.

2) Concentrations en sels biliaires fotaux. - Les variations journalières des concentrations en sels biliaires, déterminées à une heure donnée pour le même animal, sont plus importantes qu'elles ne l'étaient pour les volumes (tabl. 4). En revanche, l'évolution de ces concentrations, comparée à celle des débits biliaires, est plus homogène dans le temps : minima ef maxima s'observent sur des périodes plus étroites pour l'ensemble des porcs expérimentés.

TABLEAU 4

Étude de l'excrétion biliaire chez le porc

Variations des concentrations en sels biliaires (en pourcentage des valeurs obtenues à $9 \mathrm{~h}$ ) Amplitude des maxima et du minimum en fonction des heures de distribution des repas ( $9 \mathrm{~h}$ et $16 \mathrm{~h}$ )

\begin{tabular}{|c|c|c|c|c|c|c|c|c|c|c|c|c|c|}
\hline \multirow{3}{*}{ Porc } & \multicolumn{4}{|c|}{$1^{\text {er }}$ maximum } & \multicolumn{4}{|c|}{ Minimum } & \multicolumn{4}{|c|}{$2^{e}$ maximum } & \multirow{3}{*}{$\begin{array}{c}\text { Moyennes } \\
\text { observées } \\
\text { à } 9 \mathrm{~h} \\
\text { (base } 100 \\
\mathrm{mg} / \mathrm{ml}\end{array}$} \\
\hline & \multirow{2}{*}{$\begin{array}{c}\text { Heure } \\
\text { du } \\
\text { jour }\end{array}$} & \multicolumn{3}{|c|}{ Concentrations } & \multirow{2}{*}{$\mid \begin{array}{c}\text { Heure } \\
\text { du } \\
\text { jour }\end{array}$} & \multicolumn{3}{|c|}{ Concentrations } & \multirow{2}{*}{$\begin{array}{c}\text { Heure } \\
\text { du } \\
\text { jour }\end{array}$} & \multicolumn{3}{|c|}{ Concentrations } & \\
\hline & & $\bar{x}\left({ }^{1}\right)$ & $\overline{s X}\left({ }^{2}\right)$ & $C V\left({ }^{3}\right)$ & & $\bar{x}$ & $s \bar{x}$ & $\mathrm{CV}$ & & $\bar{x}$ & $s \bar{x}$ & $\mathrm{CV}$ & \\
\hline 1 & $\begin{array}{l}12 \mathrm{~h} \\
(12)\end{array}$ & 236 & 15 & 22 & $\begin{array}{c}16 \mathrm{~h} \\
(11)\end{array}$ & 165 & 18 & 37 & $\begin{array}{c}19 \mathrm{~h} \\
(10)\end{array}$ & 247 & 42 & 54 & 9,6 \\
\hline 2 & $\begin{array}{l}13 \mathrm{~h} \\
(12)\end{array}$ & 353 & 46 & 45 & $\begin{array}{l}16 \mathrm{~h} \\
(12)\end{array}$ & 184 & 26 & 49 & $\begin{array}{c}19 \mathrm{~h} \\
(5)\end{array}$ & 397 & 88 & 49 & 8,8 \\
\hline 3 & $\begin{array}{l}11 \mathrm{~h} \\
(10)\end{array}$ & 209 & 19 & 29 & $\begin{array}{l}16 \mathrm{~h} \\
(10)\end{array}$ & 89 & 12 & 44 & $\begin{array}{l}18 \mathrm{~h} \\
(10)\end{array}$ & 154 & 20 & 40 & 17,4 \\
\hline 4 & $\begin{array}{c}10 \mathrm{~h} \\
\text { (4) }\end{array}$ & 300 & 62 & 41 & $\begin{array}{c}16 \mathrm{~h} \\
(4)\end{array}$ & 207 & 25 & 24 & $\begin{array}{c}18 \mathrm{~h} \\
(4)\end{array}$ & 286 & 50 & 35 & 11,1 \\
\hline 5 & $\begin{array}{c}14 \mathrm{~h} \\
(9)\end{array}$ & 227 & 39 & 52 & $\begin{array}{c}16 \mathrm{~h} \\
(8)\end{array}$ & 174 & 22 & 35 & $\begin{array}{c}18 \mathrm{~h} \\
(7)\end{array}$ & 265 & 36 & 36 & 15,2 \\
\hline 6 & $\begin{array}{c}12 \mathrm{~h} \\
(6)\end{array}$ & 247 & 39 & 39 & $\begin{array}{r}16 \mathrm{~h} \\
(6)\end{array}$ & 176 & 35 & 48 & $\begin{array}{c}18 \mathrm{~h} \\
(6)\end{array}$ & 366 & 54 & 36 & 6,4 \\
\hline 7 & $\begin{array}{c}11 \mathrm{~h} \\
(5)\end{array}$ & 330 & 73 & 49 & $\begin{array}{c}16 \mathrm{~h} \\
(5)\end{array}$ & 133 & 17 & 28 & $\begin{array}{c}18 \mathrm{~h} \\
(5)\end{array}$ & 297 & 76 & 57 & 8,9 \\
\hline
\end{tabular}

(1) Moyenne ; $\left(^{2}\right)$ Ecart-type de la moyenne ; $\left({ }^{3}\right)$ Coefficient de variation.

( ) Nombre d'observations effectuées pour un même animal, à l'heure indiquée. 
L'ingestion du premier repas est suivie d'une augmentation rapide des concentrations qui atteignent, entre $10 \mathrm{~h}$ ef $14 \mathrm{~h}$ selon les animaux, 208 à 353 p. 100 des valeurs prises à $9 \mathrm{~h}$. Par la suite, elles diminuent chez tous les animaux et un minimum (100 à 170 p. 100 des valeurs déterminées à 9 h) est très nettement observé à $16 h$, soit pendant l'heure qui précède l'ingestion du deuxième repas.

La période qui suit ce deuxième repas est marquée par une augmentation immédiate des concentrations; les maxima surviennent très régulièrement entre $18 \mathrm{~h}$ et $19 \mathrm{~h}$ pour tous les animaux et représentent 155 à 397 p. 100 des valeurs prises à $9 \mathrm{~h}$.

Dans les 2 h consécutives à ce deuxième pic postprandial, les concentrations diminuent très fortement, puis tendent progressivement vers leurs valeurs minimales entre $6 \mathrm{~h}$ et $9 \mathrm{~h}$ du matin.

3) Excrétion des sels biliaires totaux. - On retrouve, pour ce dernier critère, le profil général décrit précédemment, affecté des écarts-types les plus élevés (†abl. 5).

\section{TABLEAU $V$}

\section{Éfude de l'excrétion biliaire chez le porc}

Variations des quantités de sels biliaires excrétées (en pourcentage des valeurs obtenues à 9 h) Amplitude des maxima ef du minimum en fonction des heures de distribution des repas ( $9 \mathrm{~h}$ ef $16 \mathrm{~h}$ )

\begin{tabular}{|c|c|c|c|c|c|c|c|c|c|c|c|c|c|}
\hline \multirow{3}{*}{ Porc } & \multicolumn{4}{|c|}{ 1er maximum } & \multicolumn{4}{|c|}{ Minimum } & \multicolumn{4}{|c|}{$2^{\mathrm{e}}$ maximum } & \multirow{3}{*}{$\begin{array}{l}\text { Moyennes } \\
\text { observées } \\
\text { à } 9 \mathrm{~h} \\
\text { (base } 100) \\
(\mathrm{mg} / \mathrm{h})\end{array}$} \\
\hline & \multirow{2}{*}{$\begin{array}{c}\text { Heure } \\
\text { du } \\
\text { jour }\end{array}$} & \multicolumn{3}{|c|}{$\begin{array}{c}\text { Quantités de sels } \\
\text { biliaires }\end{array}$} & \multirow{2}{*}{$\begin{array}{c}\text { Heure } \\
\text { du } \\
\text { jour }\end{array}$} & \multirow{2}{*}{$\frac{\text { de }}{x}$} & \multicolumn{2}{|c|}{$\begin{array}{l}\text { Quantités } \\
\text { sels biliaires }\end{array}$} & \multirow{2}{*}{$\begin{array}{c}\text { Heure } \\
\text { du } \\
\text { jour }\end{array}$} & \multicolumn{3}{|c|}{$\begin{array}{c}\text { Quantités } \\
\text { de sels biliaires }\end{array}$} & \\
\hline & & $\bar{x}(1)$ & $s \bar{X}\left({ }^{2}\right)$ & $C V\left({ }^{3}\right)$ & & & $s \vec{x}$ & $\mathrm{CV}$ & & $\bar{x}$ & $s \bar{x}$ & $\mathrm{CV}$ & \\
\hline 1 & $\begin{array}{c}13 \mathrm{~h} \\
(9)\end{array}$ & 464 & 109 & 67 & $\begin{array}{c}15 \mathrm{~h} \\
(7)\end{array}$ & 359 & 116 & 86 & $\begin{array}{c}17 \mathrm{~h} \\
(8)\end{array}$ & 424 & 89 & 50 & 384 \\
\hline 2 & $\begin{array}{c}13 \mathrm{~h} \\
(8)\end{array}$ & 297 & 46 & 44 & $\begin{array}{c}16 \mathrm{~h} \\
(8)\end{array}$ & 256 & 61 & 67 & $\begin{array}{c}17 \mathrm{~h} \\
(8)\end{array}$ & 387 & 84 & 61 & 725 \\
\hline 3 & $\begin{array}{c}10 \mathrm{~h} \\
\text { (4) }\end{array}$ & 297 & 70 & 53 & $\begin{array}{c}17 \mathrm{~h} \\
(5)\end{array}$ & 174 & 41 & 58 & $\begin{array}{c}18 \mathrm{~h} \\
(5)\end{array}$ & 196 & 33 & 41 & 984 \\
\hline 4 & $\begin{array}{c}12 \mathrm{~h} \\
\text { (4) }\end{array}$ & 457 & 111 & 73 & $\begin{array}{c}16 \mathrm{~h} \\
(4)\end{array}$ & 300 & 73 & 43 & $\begin{array}{c}18 \mathrm{~h} \\
\text { (4) }\end{array}$ & 387 & 89 & 65 & 935 \\
\hline 5 & $\begin{array}{c}14 h \\
(6)\end{array}$ & 317 & 87 & 67 & $\begin{array}{c}16 \mathrm{~h} \\
(6)\end{array}$ & 247 & 61 & 60 & $\begin{array}{c}18 \mathrm{~h} \\
\text { (5) }\end{array}$ & 447 & 134 & 67 & 1328 \\
\hline 6 & $\begin{array}{c}13 \mathrm{~h} \\
(6)\end{array}$ & 245 & 39 & 38 & $\begin{array}{c}16 \mathrm{~h} \\
(6)\end{array}$ & 215 & 48 & 54 & $\begin{array}{c}19 \mathrm{~h} \\
(6)\end{array}$ & 307 & 91 & 72 & 394 \\
\hline 7 & $\begin{array}{c}15 \mathrm{~h} \\
\text { (3) }\end{array}$ & 323 & 55 & 29 & $\begin{array}{c}16 \mathrm{~h} \\
(3)\end{array}$ & 188 & 27 & 25 & $\begin{array}{c}18 \mathrm{~h} \\
(3)\end{array}$ & 288 & 62 & 37 & 232 \\
\hline
\end{tabular}

(1) Moyenne ; $\left(^{2}\right)$ Ecart-type de la moyenne ; $\left(^{3}\right)$ Coefficient de variation.

( ) Nombre d'observations effectuées pour un même animal, à l'heure indiquée.

Ainsi, la quantité de sels biliaires excrétés augmente rapidement dans les heures qui suivent la distribution du premier repas. Les maxima sont observés entre $10 \mathrm{~h}$ et $15 \mathrm{~h}$, selon les animaux. L'amplitude de cette vague est la plusimportante, comparativement aux volumes et aux concentrations : à l'exception d'un animal (porc 6), elle atteint ou dépasse 300 p. 100 des valeurs prises à 9 h. L'évolution des valeurs 
observées durant l'heure postprandiale chez le porc 6, reflète un niveau secrétoire et une concentration en sels biliaires particulièrement faibles au cours de cette période. Les quantités de sels biliaires excrétés diminuent légèrement jusqu'à $16 \mathrm{~h}$ (188 à 256 p. 100 des valeurs déterminées à 9 h) excepté pour le Porc 3 chez qui cette diminution (174 p. 100) se poursuit jusqu'à $17 \mathrm{~h}$.

L'ingestion du second repas entraîne une augmentation des quantités de sels biliaires excrétés, atteignant, entre 17 et $19 \mathrm{~h}$, des maxima s'élevant généralement à plus de 300 p. 100 des valeurs prises à $9 \mathrm{~h}$.

Entre $6 \mathrm{~h}$ et $9 \mathrm{~h}$ du matin, l'excrétion de sels biliaires totaux devient minimale.

\section{Discussion.}

L'examen des coefficients de variation des moyennes calculées pour l'ensemble des porcs met en évidence l'existence d'une forte variabilité entre animaux. Ceci esł classiquement observé dans toutes les études réalisées sur l'animal fistulé (Corring, 1974 ; Laplace et Ouaissi, 1977 ; Sambrook, 1978). Compte tenu de cette variabilité, les résultats montrent en premier lieu que la quantité de sels biliaires excrétés par $24 \mathrm{~h}$ chez le Porc est de l'ordre de 35 à $40 \mathrm{~g}$.

A notre connaissance, seuls Egger et al. (1974) ont mesuré le niveau de l'excrétion des sels biliaires chez le Porc, mais les animaux utilisés étaient cholécystectomisés et la circulation entérohépatique maintenue artificiellement par des injections intraduodénales de taurocholate de sodium. Ceci peut expliquer les valeurs inférieures, rapportées par ces auteurs (15,5 à 23,2 g/24 h), pour des animaux de poids vif identique. Si l'on compare les quantités moyennes de sels biliaires excrétés par $24 \mathrm{~h}$ et par $\mathrm{kg}$ de poids vif chez différentes espèces, il apparaît que la valeur obtenue chez le Porc $(830 \mathrm{mg})$ est très largement supérieure à celles déterminées chez l'Homme : 340 mg (Miettinen, 1973) ; chez le Mouton : $200 \mathrm{mg}$ (Heath, Caple ef Redding, 1970) et chez le Chien : $400 \mathrm{mg}$ (Wheeler et Ramos, 1960). Ces différences, apparemment liées à l'espèce étudiée, pourraient s'expliquer principalement par le recyclage artificiel de sels ou acides biliaires d'origine exogène, pratiqué par ces auteurs. Chez le Singe, par contre (Dowling et al., 1970), la bile recueillie étant entièrement retournée à l'animal par voie duodénale, la quantité de sels biliaires excrétés $(880 \mathrm{mg} / \mathrm{kg} / 24 \mathrm{~h})$ est voisine de celle observée chez le Porc, dans le présent travail.

En ce qui concerne les volumes biliaires, également rapportés au poids vif, ils sont encore supérieurs chez le Porc: $46 \mathrm{ml} / \mathrm{kg} / 24 \mathrm{~h}$ contre $9 \mathrm{ml} / \mathrm{kg} / 24 \mathrm{~h}$ chez l'Homme (Miettinen, 1973), $21 \mathrm{ml} / \mathrm{kg} / 14 \mathrm{~h}$ chez le Mouton (Heath, Caple et Redding, 1970 ) et $10 \mathrm{ml} / \mathrm{kg} / 24 \mathrm{~h}$ chez le Chien (Wheeler et Ramos, 1960). Cette importante quantité de bile exportée par le Porc a également été observée par Laplace ef Ouaissi (1977) et Sambrook (1978). De plus, les valeurs rapportées $(2143 \mathrm{ml} / 24 \mathrm{~h}$ ) pour des animaux de poids comparable par Laplace et Ouaissi (1977) et obtenues lors de réintroductions duodénales, concordent parfaitement avec celles enregistrées dans cette étude. Il apparaît cependant que le site de réintroduction de la bile puisse être à l'origine de modifications du volume biliaire exporté par $24 \mathrm{~h}$, puisque Laplace ef Ouaissi (1977) et Sambrook (1978) notent un niveau d'excrétion supérieur lorsque la bile est restituée au niveau du bas cholédoque. Selon Laplace ef Ouaissi (1977), 
l'exclusion fonctionnelle du sphincter d'Oddi, due à la réintroduction intestinale de la bile, pourrait entraîner la suppression de la fonction vésiculaire. Le débit cholédocien, mesuré dans ces conditions, serait alors assimilé à un débit de bile hépatique. Il existerait en effet (Laplace, 1976) un contrôle nerveux de l'évacuation vésiculaire d'origine oddienne. Si on admet cette hypothèse, comment expliquer l'excrétion d'une bile fortement concentrée en sels biliaires, observée dans notre étude durant les périodes postprandiales? Deux hypothèses peuvent être envisagées : ou bien la technique des restitutions duodénales de la bile a affecté la fonction vésiculaire sans la supprimer complètement ; ou bien, comme le suggèrent Kimball, Pertsemlidis ef Panveliwalla (1976) chez l'Homme, l'augmentation postprandiale en l'absence de vésicule biliaire provient d'une décharge de sels biliaires stockés dans le foie ou à un autre niveau de la circulation entérohépatique. Cette deuxième éventualité serait cependant peu probable puisque, selon Wakin (1971) et Edmunds et al. (1971), l'arbre biliaire est un réservoir insignifiant. La première hypothèse nous paraîr plus vraisemblable, d'autanf plus que les relations nerveuses entre l'Oddi ef la vésicule biliaire, soulignées par Laplace (1976) ne sont pas les seules à contrôler l'évacuation vésiculaire. L'excitation du nerf vague entraîne la contraction de la vésicule chez le Chien (Pissidis, Nyhus er Bombeck, 1973), chez le Singe (Tanturi et lvy, 1938) et chez l'Homme (Balwin ef al., 1966). De plus, l'arrivée du substrat alimentaire dans la lumière intestinale, provoque chez le Chien (Fritz et Brooks, 1963) une libération de cholécystokinine, responsable également des contractions vésiculaires. Bien que la réactivité de la vésicule du Porc à cette hormone n'ait pas été vérifiée, il apparaît dans cette étude que les fortes concentrations postprandiales coïncident avec l'évacuation gastrique, particulièrement importante chez le Porc pendant les 2 premières heures consécutives au repas.

Si l'on considère l'évolution dans le temps des concentrałions et volumes biliaires, il ressort que les concentrations sont principalement responsables de l'augmentation des quantités de sels biliaires excrétés pendant les heures qui suivent les prises de nourriture. Corring, Aumaitre et Rérat (1972) ont également observé chez le Porc une augmentarion de l'excrétion des enzymes pancréatiques pendant les périodes digestives. L'évolution des quantités de sels biliaires en fonction du rythme d'alimentation a été décrite chez le Chien (Jones et Grossman, 1969) et le Rat (Hoet Drummond, 1975). Chez l'Homme, La Russo ef al. (1974) mesurent l'augmentation des acides biliaires sanguins en réponse à 3 repas liquides distribués à des sujets volontaires bien portants et montrent que la quantité d'acides biliaires circulants est maximale $2 \mathrm{~h}$ environ après chaque repas. De même Brunner ef al. (1974), par dosage des acides biliaires dans les contenus intestinaux, enregistrent une augmentation immédiate des acides biliaires présents après chaque perfusion avec un repas liquide.

Par contre, en ce qui concerne les volumes de bile émise, il n'apparaît pas de relation immédiate entre les périodes digestives et les maxima enregistrés. L'augmentation volumétrique de la sécrétion biliaire ne survient que 2 à $3 \mathrm{~h}$ en moyenne après chacun des 2 repas. Les observations rapportées par Sambrook (1978) sur Porcs nourris d'un régime de céréales, concordent avec ces derniers résultats ; selon cet auteur, il existe 2 périodes durant lesquelles l'excrétion de la bile est élevée : de $12 \mathrm{~h}$ à $15 \mathrm{~h}$, après le repas de $9 \mathrm{~h}$, ef de $20 \mathrm{~h}$ à $24 \mathrm{~h}$, après le repas de $15 \mathrm{~h}$. Par contre le débit biliaire n'est pratiquement pas affecté par l'ingestion d'un régime semi- 
purifié. Laplace et Ouaissi (1977), ne signalent pas d'augmentation de l'excrétion de bile après la distribution d'un régime à base de céréales. D'une manière générale, les quantités de bile émise au cours du nycthémère présentent, selon ces auteurs, des fluctuations beaucoup moins nettes que celles enregistrées ici. Il est probable que le niveau constant de réintroduction de la bile, appliqué par Laplace et Ouaissi (1977) tout au long du nycthémère, ait nivelé le profil de l'évolution de l'excrétion de la bile. Cependant les ajustements manuels réalisés dans le travail rapporté ici demeurent forcément approximatifs et il conviendrait d'entreprendre la recherche d'un rythme nycthéméral véritable, dans des conditions de réintroduction de la bile rigoureusement calquée sur le débit cholédocien.

Reçu en juin 1978.

Accepté en septembre 1978.

\section{Références}

BALWIN J., HEER F. W., ALBO R., PELOSO O., RUBY L., SILEN W., 1966. Effect of vagus nerves stimulation on hepatic secretion of bile in human subjects. Am. J. Surg., 3, 66-69.

BERGSTROM S., BORGSTROM B., 1955. Some aspects of the intestinal absorption of fats, 353393. In HOLMAN R. T., LUNDBERG W. O., MALKIN T., Progress in the chemistry of fats and other lipids. Pergamon Press, London.

BORTZ W. M., STEELE L. A., 1973. Synchronization of hepatic cholesterol synthesis cholesterol, and bile acid content, fatty acid synthesis and plasma free fatty acids levels in the fed and fasted rat. Biochem. biophys. Acta, 306, 85-94.

BRUNNER H., NORTHFIELD T. C., HOFMANN A. F., GO V. L. W., SUMMERSKILL W. H.J., 1974. Gastric emptying and secretion of bile acids, cholesterol and pancreatic enzymes during digestion. Mayo clin. Proc., 49, 851-860.

CORRING T., 1974. Régulation de la sécrétion pancréatique par rétro-action négative chez le porc. Ann. Biol. anim. Bioch. Biophys., 14, 487-498.

CORRING T., AUMAITRE A., RÉRAT A., 1972. Fistulation permanente du pancréas exocrine chez le porc. Application : réponse de la sécrétion pancréatique au repas. Ann. Biol. anim. Bioch. Biophys., 12, 109-124.

DOWLING R. H., MACK E., SMALL D. M., PICOTT J., 1970. Effects of controlled interruption of the enterohepatic circulation of bile salts by biliary diversion and by ileal resection on bile salt secretion, synthesis, and pool size in Rhesus monkey. J. clin. Inv., 49, 232-242.

EDMUNDS R., KATZ S., GARCIANO V., FINBY N., 1971. The common duct after cholecystectomy : interval report. Arch. Surg., 103, 79-81.

EGgER G., KUTZ K., STREBEL H., BIRCHER J., WEBER M., SCHOLL E., PREISIG R., 1974. Bile formation in the intact pig. Am.J. vet. Res., 35, 1203-1208.

FORSGREN E., 1928. On the relationship between the formation of bile and glycogen in the liver of rabbit. Skand. Arch. Physiol., 53, 137-151.

FRITZ M. E., BROOKS F. P., 1963. Control of bile flow in the cholecystectomized dog. Am. J. Physiol., 204, 825-828.

HEATH T., CAPLE I. W., REDDING P. M., 1970. Effect on the entherohepatic circulation of bile salts on the flow of bile and its content of bile salts and lipids in sheep. Quart. J. expt. Physiol., 55, 93-103.

HO K. J., DRUMMOND J. L., 1975. Circadian rythm of biliary excretion and its control mechanisms in rats with chronic biliary drainage. Am. J. Physiol., 229, 1427-1437.

HOFMANN A. F., 1964. The function of bile salts in fat absorption. M. D. Thesis. Lund, Sweden, Univ. of Lund.

HOLMGREN H., 1931. Beitrag zur Kenntnis von der Leber Funktion. Z. mikrosk.- anat. Forsch., 24, 632-642.

HOLMQUIST A. G., 1931. Beitrage zur Kenntnis der 24 Stündigen Rhythmik der Leber Z. mikrosk.anat. Forsch., 25, 30-43. 
HURLOCK B., TALALAY P., 1956. Enzymatic estimation of urinary steroids. Proc. Soc. expt. Biol. Med., 93, 560-564.

HURLOCK B., TALALAY P., 1957. Principles of the enzymatic measurement of steroids. J. biol. Chem., 227, 37-52.

JONES R. S., GROSSMAN M. I., 1969. The choleretic response to feeding in dogs. Proc. Soc. expt. Biol. Med., 132, 708-711.

KIMBall A., PERTSEmLIDIS D., PANVELIWALla D., 1976. Composition of biliary lipids, and kinetics of bile acids after cholecystectomy in man. Dig. Dis., 21, 776-781.

LAPLACE J. P., 1976. L'excrétion biliaire chez le porc. 2. Electromyographie et dynamique de l'excrétion de bile. Rec. Med. vét., 152, 401-411.

LAPLACE J. P., OUAISSI M. A., 1977. L'excrétion biliaire chez le porc. Influence des repas et rôle éventuel de récepteurs oddiens dans le contrôle du débit cholédocien. Ann. Zootech., 26, 595-613.

LA RUSSO N. F., KORMAN M. G., hOFFMAN N. E., HOFMANN A. F., 1974. Dynamics of the enterohepatic circulation of bile acids. Postprandial serum concentrations of conjugates of cholic acid in heatlth, cholecystectomized patients and patients with bile acid malabsorption. N. Engl. J. Med., 291, 689-692.

MARTIN E., SCICLOUNOFF F., 1934. Rythme nycthéméral de la glycorégulation et sensibilité à l'insuline. Presse méd., 42, 1936-1938.

MIETTINEN T. A., 1973. Clinical implications of bile acid metabolism in man, 191-247. In NAIR P. P., KRITCHEVSKY D., The bile acids, Vol. 2, Plenum Press, New-York-London.

PISSIDIS A. G., NYHUS L. M., BOMBECK C. T., 1973. Neurohumoral control of bile secretion in the cholecystectomized concious dog. Surgery, 74, 122-129.

SAMBROOK I. E., 1978. Studies on the digestion and absorption of carbohydrates and fats and on the flow and composition of bile in the growing pig. Ph. D. Thesis. Reading Univ.

TALALAY P., DOBSON M.-M., 1953. Purification and properties of a $\beta$-hydroxysteroid dehydrogenase. J. biol. Chem., 205, 823-837.

TANTURI C. A., IVY A. C., 1938 . On the existence of secrefory nerves in the vagi, and the reflex excitation and inhibition of bile secretion. Am. J. Physiol., 121, 270-283.

WAKIN K. G., 1971. Passive role of the bile duct system in the delivery of bile into the intestine. Surg. Gynecol. Obstet., 133, 826-829.

WHEELER H. O., RAMOS O. L., 1960 . Determinants of the flow and composition of bile in the unanesthetized dog during constant infusions of sodium taurocholate. J. clin. Inv., 30, 161-169. 